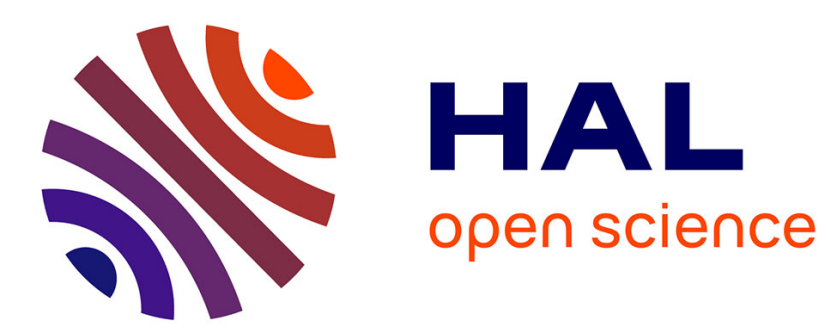

\title{
Toward a coherent Monte Carlo simulation of CVA
}

Lokman Abbas-Turki, Ayech Bouselmi, Mohammed Mikou

\section{To cite this version:}

Lokman Abbas-Turki, Ayech Bouselmi, Mohammed Mikou. Toward a coherent Monte Carlo simulation of CVA. 2013. hal-00873200v1

\section{HAL Id: hal-00873200 https://hal.science/hal-00873200v1}

Preprint submitted on 15 Oct 2013 (v1), last revised 12 Dec 2013 (v2)

HAL is a multi-disciplinary open access archive for the deposit and dissemination of scientific research documents, whether they are published or not. The documents may come from teaching and research institutions in France or abroad, or from public or private research centers.
L'archive ouverte pluridisciplinaire HAL, est destinée au dépôt et à la diffusion de documents scientifiques de niveau recherche, publiés ou non, émanant des établissements d'enseignement et de recherche français ou étrangers, des laboratoires publics ou privés. 


\title{
Toward a coherent Monte Carlo simulation of CVA
}

\author{
L. A. Abbas-Turki ${ }^{*}$ A. I. Bouselmi ${ }^{\dagger}$ and M. A. Mikou \\ *TU Berlin, Stochastik und Finanzmathematik, \\ Building MA, Str. des 17. Juni 136, 10623 Berlin, Germany \\ †Université Paris-Est, Laboratoire d'Analyse et de Mathématiques Appliquées, \\ Champs-sur-Marne, 77454 Marne-la-Vallée Cedex2, France \\ $\ddagger$ EISTI, Laboratoire de Mathématiques, \\ avenue du Parc, 95011 Cergy-Pontoise Cedex, France
}

\begin{abstract}
This paper is devoted to the simulation of the Credit Valuation Adjustment (CVA) using a pure Monte Carlo technique with Malliavin Calculus (MCM). The procedure presented is based on a general theoretical framework that includes a large number of models as well as various contracts, and allows both the computation of CVA and its sensitivity with respect to the different assets. Moreover, we provide the expression of the backward conditional density of assets vector that can be simulated off-line in order to reduce the variance of the CVA estimator. Regarding computational aspects, both complexity and accuracy are studied for MCM and regression methods and compared to the square Monte Carlo benchmark.
\end{abstract}

\section{Introduction}

After the 2007 economic crisis, several laws were issued for better financial regulation. Among the most important measures are those taken at Basel III that include the calculation of the CVA (Credit Valuation Adjustment) as an important part of the prudential rules. In a financial transaction between a party $A$ that has to pay another party $B$ some amount $V$, the CVA value is the price of the insurance contract that covers the default of party $A$ to pay the whole sum $V$. In other words, in the absence of arbitrage opportunities, the CVA is the value of liquid products that must be saved to deal with counterparty default (see $[9,10])$.

Formally speaking, the CVA is given by the following equality

$$
\begin{aligned}
\mathrm{CVA}_{t, T} & =(1-R) E_{t}\left(V_{\tau}^{+} 1_{t<\tau \leq T}\right) \\
& =(1-R) \int_{t}^{T} E_{t}\left(V_{u}^{+} \mid \tau=u\right) P_{\tau}(d u),
\end{aligned}
$$

where $R$ (assumed equal to zero in this paper) is the recovery to make on the portfolio if the counterparty defaults, $E_{t}$ denotes the conditional expectation knowing 
all the available information at $t, V_{t}$ is the process of the value exposure to the counterparty, $\tau$ is the random default time of the counterparty, $T$ is the protection time horizon and the positive part function is denoted either by ${ }^{+}$or $_{+}$.

What makes difficult the numerical approximation of (1) is the expression of $V_{t}$ that does not only includes assets, but also European contracts and American contracts. Said differently, once the stochastic model of the assets is fixed, one needs to simulate contracts before simulating the value of the CVA. The approximation of (1) is performed through three steps: First, simulating the assets $S_{t}=\left(S_{t}^{1}, \ldots, S_{t}^{d}\right)$ trajectories, then simulating the contracts trajectories to get $V_{t}$ trajectories as a sum over the whole exposure:

$$
V_{t}=\sum_{i e} \phi_{i e}^{e x p}\left(S_{t}\right)+\sum_{i i} \phi_{i i}^{e u i}\left(S_{t}\right)+\sum_{i d} \phi_{i d, t}^{e u d}\left(S_{t}\right)+\sum_{i a} \phi_{i a, t}^{a m}\left(S_{t}\right),
$$

where $i e, i i, i d$ and $i a$ are the exposure indices and:

$\phi^{\exp }$ is an explicit function that represents pure assets transaction, for example: $\phi^{\exp }\left(S_{t_{k}}\right)=S_{t_{k}}^{1}-S_{t_{k}}^{2}$.

$\phi^{e u i}$ is a path-independent European contract. It is a contract involving only the simulation of the assets $S_{t}$ at $t=T$ and whose expression is given thanks to an explicit payoff function $f^{e u i}$ by

$$
\phi^{\text {eui }}\left(S_{t_{k}}\right)=E\left(f^{e u i}\left(S_{T}\right) \mid S_{t_{k}}\right), \text { with } t_{k} \in[0, T],
$$

for example: $f^{e u i}\left(S_{T}\right)=\left(S_{T}^{1}-S_{T}^{2}\right)_{+}$.

$\phi_{t}^{\text {eud }}$ is a path-dependent European contract. It is a contract involving the simulation of the whole discretized path of $S_{t}$ at $t \in\left\{t_{0}, t_{1}, \ldots, T\right\}$ and whose expression is given thanks to a path-dependent payoff function $f_{t}^{\text {eud }}$, at each time $t_{k}$ by

$$
\phi_{t_{k}}^{e u d}\left(S_{t_{k}}\right)=E\left(f_{t_{k}}^{e u d}\left(S_{t_{k+1}}\right) \mid S_{t_{k}}\right),
$$

for example: $f_{t_{k}}^{e u d}\left(S_{t_{k+1}}\right)=\left(\max _{i=0, . ., k} S_{t_{i}}^{1} \vee S_{t_{k+1}}^{1}-S_{t_{k+1}}^{2}\right)_{+}$where $\vee$ is the maximum operator. In the previous example, the dependence of the payoff according to the information available at time $t_{k}$ is illustrated by $\max _{i=0, . ., k} S_{t_{i}}^{1}$.

$\phi_{t}^{a m}$ is an American contract. It is a contract that depends on the assets path through an optimal stopping problem implemented by the dynamic programming algorithm

$$
\phi_{t_{k}}^{a m}\left(S_{t_{k}}\right)=f\left(S_{t_{k}}\right) \vee E\left(\phi_{t_{k+1}}^{a m}\left(S_{t_{k+1}}\right) \mid S_{t_{k}}\right)
$$

with $f$ an explicit payoff that generally does not depend on the asset path.

Without loss of generality, assuming $t=0$ and $R=0$ in (1), the last step of approximating $\mathrm{CVA}_{0, T}$ is based on a time discretization, to get

$$
\mathrm{CVA}_{0, T} \approx \sum_{k=0}^{N-1} E\left(V_{t_{k}}^{+} 1_{\tau \in\left(t_{k}, t_{k+1}\right]}\right)
$$


and $N$ must be smaller or equal to the number of time steps used to approximate the trajectories of the assets.

The last point that has to be specified is the model used for the default time $\tau$ and how it should be related to the dynamic of $V_{t}$. In this paper, the two main families of modeling default time are studied: i) The reduced form family, ii) The structural family. For each family, a specific model involving dependence between the exposure and the default time (so called WWR/RWR) is considered with its associated expressions of the computation of $\mathrm{CVA}_{0, T}$ and $\partial_{S_{0}^{i}} \mathrm{CVA}_{0, T}$, where $S_{0}^{i}$ is the $i^{\text {th }}$ spot price. The latter quantities will be expressed only as a function of the exposure and its gradient vector.

The common point between the expressions (3), (4) and (5) is the computation of a conditional expectation that should be simulated before the approximation (6). In this paper, we reexpress both the conditional expectation involved and its gradient using Malliavin Calculus. Denoting $E\left(f\left(S_{t_{k+1}}\right) \mid S_{t_{k}}\right)=\varphi\left(S_{t_{k}}\right)$, we aim at computing

$$
\varphi(x)=E\left(f\left(S_{t_{k+1}}\right) \mid S_{t_{k}}=x\right) \text { and } \partial_{x_{i}} \varphi(x) \text { with } x_{i} \in\left\{x_{1}, \ldots, x_{d}\right\} .
$$

Inspired from papers $[2,3]$ dedicated to American contracts, we rewrite (7) as a quotient of expectations that can be simulated by Monte Carlo. Going beyond the latter result, we express the backward conditional density and its gradient as a quotient of expectations that can be simulated by Monte Carlo. When this density and its gradient are known off-line for some trajectories (after discretization), then the values given in (7) can be efficiently approximated for any path-dependent or path-independent function $f$.

One could wonder why it is more advantageous to use Malliavin Calculus with Monte Carlo simulation (MCM) when compared to the direct use of a square ${ }^{1}$ Monte Carlo simulation. We will see that MCM is justified by its computational complexity which is smaller than square Monte Carlo, in particular for the CVA that involves American contracts. Moreover, in contrast to regression methods used in [10], we will justify that MCM is a nonparametric method whose accuracy depends only on the number of simulated trajectories. Consequently, one can increase "indefinitely" the accuracy by simulating more trajectories and using more computational resources thanks to an efficient parallel implementation.

The easy adaptability to various models is a key advantage of our procedure, then we present in Section 2 a brief summary of the different models that can be used. In Section 3, we provide, on the one hand, the expression of the conditional expectation (7) as well as its partial derivative and we introduce, on the other hand, the value of the backward conditional density that can be simulated off-line to speedup the convergence of the CVA estimator. In Section 4, we compare the computational complexity of MCM with the method that involves regressions and with the square Monte Carlo method used as a benchmark. In addition to the MCM

\footnotetext{
${ }^{1}$ This is a two levels Monte Carlo simulation, one for simulating assets trajectories and another one for simulating contracts trajectories. We refer the reader to Section 4 for a detailed explanation of the term square.
} 
general framework, its adaptability to parallel architecture and its good accuracy studied in Section 5 suffice to justify its use as a real alternative to regression methods.

\section{Model families}

In this Section, we present the general modelling framework of asset prices and counterparty default for which MCM could be applied. We also express $\mathrm{CVA}_{0, T}$ and $\partial_{S_{0}^{i}} \mathrm{CVA}_{0, T}$ using $V_{t}$ and $\partial_{S_{0}^{i}} V_{t}$ whose values are given by (2) and

$$
\begin{aligned}
\partial_{S_{0}^{i}} V_{t}=\sum_{i e} \partial_{S_{0}^{i}} \phi_{i e}^{e x p}\left(S_{t}\right) & +\sum_{i i} \partial_{S_{0}^{i}} \phi_{i i}^{e u i}\left(S_{t}\right)+\sum_{i d} \partial_{S_{0}^{i}} \phi_{i d, t}^{e u d}\left(S_{t}\right) \\
& +\sum_{i a} \partial_{S_{0}^{i}} \phi_{i a, t}^{a m}\left(S_{t}\right) .
\end{aligned}
$$

The value of each $\phi$ term in (2) and its derivative in (8) has the general formulation (7) given by $\varphi$ and its derivative which are both computed thanks to the results presented in Section 3. Without loss of generality, we remind that we assume $R=0$ in (1).

Let $T$ be the protection time horizon, $(\Omega, \mathcal{F}, P)$ a probability space on which we define a $d$-dimensional standard Brownian motion $W=\left(W^{1}, \ldots, W^{d}\right)$ and $\mathbb{F}=$ $\left\{\mathcal{F}_{s}\right\}_{s \leq T}$ the $P$-completion of the filtration generated by $W$ until $T$. We denote by $S_{t}$ the vector of asset prices $S_{t}^{1}, \ldots, S_{t}^{d}$. which are the solutions of the following stochastic differential equations

$$
\frac{d S_{t}^{i}}{S_{t}^{i}}=r_{i} d t+\sum_{j=1}^{i} \sigma_{i j}(t) d W_{t}^{j}, \quad S_{0}^{i}=z_{i}, \quad i=1, . ., d,
$$

where $r_{i}$ are constants and $\sigma(t)=\left\{\sigma_{i j}(t)\right\}_{1 \leq i, j \leq d}$ is a deterministic triangular matrix $\left(\left\{\sigma_{i j}(t)\right\}_{i<j}=0\right)$. We suppose that the matrix $\sigma(t)$ is invertible, bounded and uniformly elliptic which ensures the existence of the inverse matrix $\rho(t)=\sigma^{-1}(t)$ and its boundedness. Dynamics (9) is widely used for equity models, HJM interest rate models and variance swap models. One should note that in the case where the dynamics of $S$ is given by local volatility model, we can use a discretization scheme to reduce it to an SDE of type (9) on subintervals. The methodology developed in Section 3 can be extended to jump diffusion and stochastic volatility models, Indeed:

i) We can replace (9) by the following $\mathrm{SDE}$

$$
\frac{d S_{t}^{i}}{S_{t}^{i}}=r_{i} d t+\sum_{j=1}^{i} \sigma_{i j}(t) d W_{t}^{j}+d J_{t}^{i}, \quad S_{0}^{i}=z_{i}, \quad i=1, . ., d,
$$

where $J=\left(J^{1}, \ldots, J^{d}\right)$ is a jump process independent from $W$. Then the conditional expectation in (7) is given by

$$
\varphi(x)=E\left(E\left[f\left(S_{t_{k+1}}\right) \mid \sigma\left(\left(J_{u}\right)_{0 \leq u \leq t}\right), S_{t_{k}}=x\right] \mid S_{t_{k}}=x\right), x=\left(x_{1}, \ldots, x_{d}\right) .
$$


The computations performed in Section 3 can be implemented to the inner expectation in (10).

ii) We can replace (9) by the following stochastic volatility model,

$$
\frac{d S_{t}^{i}}{S_{t}^{i}}=r_{i} d t+\sum_{j=1}^{i} \sigma_{i j}\left(t, \widetilde{W}_{t}\right) d W_{t}^{j}, \quad S_{0}^{i}=z_{i}, \quad i=1, . ., d,
$$

where $\widetilde{W}$ is a multidimensional Brownian motion correlated to $W$ as it is done in [2]. Then the conditional expectation in (7) is given by

$$
\varphi(x)=E\left(E\left[f\left(S_{t_{k+1}}\right) \mid \sigma\left(\left(\widetilde{W}_{u}\right)_{0 \leq u \leq t}\right), S_{t_{k}}=x\right] \mid S_{t_{k}}=x\right), x=\left(x_{1}, \ldots, x_{d}\right)
$$

and the inner expectation in (11) and its partial derivative according to each $x_{i}$ can be computed as explained in Section 3 .

In addition to the large number of asset models that can be used, when assuming independence between $V_{u}$ and $\tau$ in (1), one has a wide choice of the counterparty default time distribution $P_{\tau}(d u)$. In fact, under the independence assumption, we have

$$
\begin{gathered}
\mathrm{CVA}_{0, T}=\int_{0}^{T} E\left(V_{u}^{+} \mid \tau\right) P_{\tau}(d u)=\int_{0}^{T} E\left(V_{u}^{+}\right) P_{\tau}(d u) \\
\partial_{S_{0}^{i}} \mathrm{CVA}=\int_{0}^{T} E\left(\partial_{S_{0}^{i}} V_{u} 1_{\left\{V_{u}>0\right\}}\right) P_{\tau}(d u) .
\end{gathered}
$$

The permutation of the differentiation $\partial_{S_{0}^{i}}$ and the expectation is possible thanks to Remark 3.1 ii).

In the following, we consider CVA models involving WWR or RWR, this implies that $V_{u}$ and $\tau$ are no longer assumed to be independent. ISDA (the International Swaps and Derivatives Association) defines Wrong Way Risk as the risk that occurs when the "exposure to a counterparty is adversely correlated with the credit quality of that counterparty", when the Right Way Risk (RWR) refers to the opposite correlation. Consequently, the choice of the counterparty default model will influence the CVA and $\partial_{S_{0}^{i}}$ CVA expressions.

Using the literature $[5,4,8,9]$, we distinguish two main ways to model the default time: i) The structural family (firm value) and ii) The reduced form (intensity) family. However, as pointed out by the authors of [11], there is no standard way to specify the dependence between the counterparty default and the exposure. Subsequently, we will only give an example for each default model with its CVA expressions. 


\subsection{CVA intensity models including WWR/RWR}

In these models, we assimilate the default time as the first jumping time of a Poisson process, where we denote by $\lambda$ its intensity. We point out that $\lambda$ can be even considered as deterministic, either constant or time dependent, and even stochastic like in Cox model. If $\lambda$ is deterministic, we have $P(\tau>\theta)=e^{-\lambda \theta}$ and more precisely

$$
P(\tau \in(t, t+d t] \mid \tau>t)=\frac{P(\tau \in(t, t+d t])}{P(\tau>t)}=\lambda d t .
$$

The same formula is true when $\lambda_{t}$ is $\mathcal{F}_{t}$-adapted by conditioning with respect to $\mathcal{F}_{t}$. Defined by

$$
\Lambda(t)=\int_{0}^{t} \lambda_{s} d s
$$

the function $\Lambda$ is commonly known as the hazard function or cumulated intensity and $\lambda$ represents the intensity or hazard rate.

When $\Lambda$ is deterministic ${ }^{2}$, the Poisson process properties imply that $\Lambda(\tau)$ is an exponential random variable with $E(\Lambda(\tau))=1$. Notice also that $\Lambda(\tau)$ is independent from the default-free market information $\mathbb{F}$. Thus we obtain,

$$
P(\tau>t)=P(\Lambda(\tau)>\Lambda(t))=e^{-\Lambda(t)}=e^{-\int_{0}^{t} \lambda_{s} d s} .
$$

If the hazard function is stochastic, we get

$$
P(\tau>t)=E\left(e^{-\int_{0}^{t} \lambda_{s} d s}\right) .
$$

\section{Specific example with its CVA and CVA sensitivity estimation}

We present the model proposed in [11], involving WWR or RWR. The intensity model is assumed to have a stochastic $\mathcal{F}_{t}$-adapted hazard rate $\lambda_{t}$. Particularly, we suppose that

$$
\lambda(t)=f\left(t, V_{t}\right),
$$

where $f$ is some "known" positive $\mathcal{C}^{1}$ function and $V_{t}$ represents the exposure at $t$. In some cases, one can take

$$
\lambda(t)=g\left(t, S_{t}\right),
$$

where $S_{t}$ is the $\mathbb{R}^{d}$ process describing the underlying asset prices. In [11], the authors assume that

$$
\lambda(t)=a(t)+\rho V_{t},
$$

where $a(t)$ is a deterministic function that is useful for the calibration and $\rho$ represents the dependence (WWR or RWR).

Assuming that we are able to estimate the process $V_{t}$ on a time discretized grid

\footnotetext{
${ }^{2}$ If $\Lambda$ is $\mathcal{F}_{t}$-adapted, we obtain the same result by conditioning with respect to $\mathcal{F}_{t}$.
} 
$\left\{t_{0}<t_{1}<t_{2}<. .<t_{n}=T\right\}$ (see Section 3 ), the value of the $\mathrm{CVA}_{0, T}$ will be given by

$$
\mathrm{CVA}_{0, T}=E\left(\sum_{k=0}^{n-1} V_{t_{k+1}}^{+} P\left(\tau \in\left(t_{k}, t_{k+1}\right] \mid \mathcal{F}_{t_{k+1}}\right)\right) .
$$

The sensitivity of the $\mathrm{CVA}_{0, T}$ according to the $i^{\text {th }}$ spot price $S_{0}^{i}$ is as follows,

$$
\begin{aligned}
\partial_{S_{0}^{i}} \mathrm{CVA}_{0, T}= & E\left(\sum_{k=0}^{n-1} \partial_{S_{0}^{i}} V_{t_{k+1}} 1_{\left\{V_{t_{k+1}}>0\right\}} P\left(\tau \in\left(t_{k}, t_{k+1}\right] \mid \mathcal{F}_{t_{k+1}}\right)\right) \\
& +E\left(\sum_{k=0}^{n-1} V_{t_{k+1}}^{+} \partial_{S_{0}^{i}} P\left(\tau \in\left(t_{k}, t_{k+1}\right] \mid \mathcal{F}_{t_{k+1}}\right)\right) .
\end{aligned}
$$

The permutation of the operator $\partial_{S_{0}^{i}}$ and the expectation is justified in Remark 3.1 ii). Regarding the derivative $\partial_{S_{0}^{i}} P\left(\tau \in\left(t_{k}, t_{k+1}\right]\right)$, we have

$$
\begin{aligned}
& \partial_{S_{0}^{i}} P\left(\tau \in\left(t_{k}, t_{k+1}\right] \mid \mathcal{F}_{t_{k+1}}\right)=\partial_{S_{0}^{i}}\left(P\left(\tau>t_{k} \mid \mathcal{F}_{t_{k+1}}\right)-P\left(\tau>t_{k+1} \mid \mathcal{F}_{t_{k+1}}\right)\right) \\
& =\partial_{S_{0}^{i}}\left(e^{-\int_{0}^{t} k f\left(s, V_{s}\right) d s}-e^{-\int_{0}^{t_{k+1}} f\left(s, V_{s}\right) d s}\right) .
\end{aligned}
$$

Using the chain rule, this derivative becomes

$$
\begin{array}{rl}
\partial_{S_{0}^{i}} & P\left(\tau \in\left(t_{k}, t_{k+1}\right] \mid \mathcal{F}_{t_{k+1}}\right) \\
=- & P\left(\tau>t_{k} \mid \mathcal{F}_{t_{k+1}}\right) \int_{0}^{t_{k}} \partial_{V_{s}} f\left(s, V_{s}\right) \partial_{S_{0}^{i}} V_{s} d s \\
& \quad+P\left(\tau>t_{k+1} \mid \mathcal{F}_{t_{k+1}}\right) \int_{0}^{t_{k+1}} \partial_{V_{s}} f\left(s, V_{s}\right) \partial_{S_{0}^{i}} V_{s} d s \\
=- & P\left(\tau \in\left(t_{k}, t_{k+1}|| \mathcal{F}_{t_{k+1}}\right) \int_{0}^{t_{k}} \partial_{V_{s}} f\left(s, V_{s}\right) \partial_{S_{0}^{i}} V_{s} d s\right. \\
& \quad+P\left(\tau>t_{k+1} \mid \mathcal{F}_{t_{k+1}}\right) \int_{t_{k}}^{t_{k+1}} \partial_{V_{s}} f\left(s, V_{s}\right) \partial_{S_{0}^{i}} V_{s} d s \\
=- & P\left(\tau \in\left(t_{k}, t_{k+1}\right] \mid \mathcal{F}_{t_{k+1}}\right) \int_{0}^{t_{k}} \rho \partial_{S_{0}^{i}} V_{s} d s+P\left(\tau>t_{k+1} \mid \mathcal{F}_{t_{k+1}}\right) \int_{t_{k}}^{t_{k+1}} \rho \partial_{S_{0}^{i}} V_{s} d s .
\end{array}
$$

Both $V_{t}$ and $\partial_{S_{0}^{i}} V_{t}$ are provided in (2) and (8).

\subsection{CVA structural models including WWR/RWR}

First introduced by Merton [13], the default time in these models is defined according to the behavior of the positive firm value process $\left(X_{t}\right)_{t \geq 0}$. Merton's example assumes that default occurs if, at the final time $T$, the firm value $X_{T}$ is below a given threshold $L$ which generally represents a promised terminal payoff.

Inspired by this model, Black and Cox proposed to modelize the default time by

$$
\tau=\inf \left\{t \geq 0 \quad \mid \quad X_{t} \leq L_{t}\right\}
$$


where

$$
L_{t}=\left\{\begin{array}{cl}
e^{-\gamma(T-t)} K & \text { if } t<T \\
L & \text { if } t=T
\end{array}\right.
$$

with $\gamma$ smaller than the risk neutral interest rate and $K \leq L$. In this situation, the "critical" threshold $L_{t}$ must not be crossed by the firm value process. For more details, we refer the reader to [6].

\section{Specific example with its $C V A$ and $C V A$ sensitivity estimation}

The dependence between the default time variable $\tau$ and the exposure $V_{t}$ is modelized thanks to the correlation between some Brownian motion $W_{t}^{0}$ that drives the process $X_{t}$ and $\left(W_{t}^{1}, . ., W_{t}^{d}\right)$ which drive the asset prices $S_{t}$.

Thus, $\mathrm{CVA}_{0, T}$ is given by

$$
\begin{aligned}
\mathrm{CVA}_{0, T} & =E\left(\sum_{k=0}^{n-1} V_{\tau}^{+} 1_{\left\{\tau \in\left(t_{k}, t_{k+1}\right]\right\}}\right) \\
& \approx E\left(\sum_{k=0}^{n-1} V_{t_{k}}^{+} 1_{\left\{\tau \in\left(t_{k}, t_{k+1}\right]\right\}}\right) .
\end{aligned}
$$

The sensitivity of $\mathrm{CVA}_{0, T}$ according to the $i^{\text {th }}$ spot price $S_{0}^{i}$ is given by

$$
\begin{aligned}
\partial_{S_{0}^{i}} \mathrm{CVA}_{0, T}= & \sum_{k=0}^{n-1} \partial_{S_{0}^{i}} E\left(V_{t_{k}}^{+} \mid \tau \in\left(t_{k}, t_{k+1}\right]\right) P\left(\tau \in\left(t_{k}, t_{k+1}\right]\right) \\
& +\sum_{k=0}^{n-1} E\left(V_{t_{k}}^{+} \mid \tau \in\left(t_{k}, t_{k+1}\right]\right) \partial_{S_{0}^{i}} P\left(\tau \in\left(t_{k}, t_{k+1}\right]\right) \\
= & \sum_{k=0}^{n-1} E\left(\partial_{S_{0}^{i}} V_{t_{k}} 1_{\left\{V_{t_{k}}>0\right\}} 1_{\left\{\tau \in\left(t_{k}, t_{k+1}\right]\right\}}\right)
\end{aligned}
$$

which is ensured by the assumption $\partial_{S_{0}^{i}} P\left(\tau \in\left(t_{k}, t_{k+1}\right]\right)=0$ and Remark 3.1 ii $)$ that allows the permutation of the operator $\partial_{S_{0}^{i}}$ and the expectation.

Both $V_{t}$ and $\partial_{S_{0}^{i}} V_{t}$ are provided in (2) and (8). Using the same argument presented in (10) and (11), the dependence according to $\tau$ is not an important issue for computations performed in Section 3. In fact, the conditional expectation (7) is equal to

$$
\varphi(x)=E\left(E\left[f\left(S_{t_{k+1}}\right) \mid \sigma\left(\left(W_{u}^{0}\right)_{0 \leq u \leq t}\right), S_{t_{k}}=x\right] \mid S_{t_{k}}=x\right), x=\left(x_{1}, \ldots, x_{d}\right)
$$

and the inner expectation can be computed as if the trajectory of $\left\{X_{u}\right\}_{0 \leq u \leq t}$ is completely known. For more details, we refer the reader to Section 5 in which a more specific example is presented. 


\section{Computing the value exposure, its sensitiv- ity and the backward conditional density}

Estimating the value exposure to the counterparty $V_{t}$ is crucial in the CVA computation. In order to calculate $V_{t}$ using (2), one has to express the conditional expectation involved in each contract. Using Malliavin calculus for American contracts pricing, this conditional expectation was expressed as a ratio of two expectations (see for example $[2,3]$ ). We aim here to adapt the previous results to the CVA problem. Moreover, we give an explicit formulation of the sensitivity with respect to the initial value of the stock price. In Section 3.2, we will be interested by rather a theoretical result that provides the value of the backward conditional density of the process (9) and of multidimensional stochastic volatility and jump diffusion models that extends (9). The backward transition probability does not depend on the payoff, then it should be computed off-line and stored to be re-used, in the same fashion as it is done in $[16,19]$.

In this section we suppose that the stock price $S$ is given by (9). To simplify the notations, we denote $H_{i}^{x}\left(S_{s}^{i}\right)=H\left(S_{s}^{i}-x_{i}\right):=1_{S_{s}^{i} \geq x_{i}}$ for the Heaviside function of the difference between the $i^{t h}$ stock and the $i^{\text {th }}$ coordinate of the positive vector $x$. Throughout this article, we assume that $g \in \mathcal{E}_{b}\left(\mathbb{R}^{d}\right)$ is a measurable function with polynomial growth

$$
\left.\mathcal{E}_{b}\left(\mathbb{R}^{d}\right)=\left\{f \in \mathcal{M}\left(\mathbb{R}^{d}\right): \exists C>0 \text { and } m \in \mathbb{N} ;|f(y)| \leq C\left(1+|y|_{d}\right)^{m}\right)\right\}
$$

where $\mathcal{M}\left(\mathbb{R}^{d}\right)$ is the set of measurable functions on $\mathbb{R}^{d}$ and $|\cdot|_{d}$ is the euclidean norm. The elements of the set $\mathcal{E}_{b}\left(\mathbb{R}^{d}\right)$ satisfy the finiteness of the expectations computed in this article. Besides, we usually use Malliavin derivative $D_{u}^{j}$ for the differentiation with respect to the $j^{\text {th }}$ Brownian motion.

\subsection{The conditional expectation value and its gradient}

We have already seen that $V_{t}$ and $\partial_{S_{0}^{i}} V_{t}$ are given by (2) and (8) where the value of each contract is expressed using (3), (4) and (5). The only point that remains to be specified is the conditional expectation and its partial derivative in (7). Theorem 3.1 deals with the latter issue, but before that we need to introduce some definitions.

Definition 3.1 We define the random variable $\Gamma_{s, t}=\Gamma_{s, t}^{1}$ and $\Gamma_{s, t}^{1}$ can be computed by the following induction scheme

$$
\Gamma_{s, t}^{d}=\pi_{s, t}^{d, d}, \Gamma_{s, t}^{k}=\Gamma_{s, t}^{k+1} \pi_{s, t}^{k, d}-\sum_{j=k+1}^{d} \int_{0}^{t} D_{u}^{j} \Gamma_{s, t}^{k+1} D_{u}^{j} \pi_{s, t}^{k, d} d u, k \in\{1, \ldots, d-1\},
$$

where $\pi_{s, t}^{k, d}$ is given by

$$
\pi_{s, t}^{k, d}=1+\sum_{j=k}^{d} \int_{0}^{t} \varphi_{j k}(u) d W_{u}^{j}, \varphi_{j k}(u)=\frac{1}{s} \rho_{j k}(u) 1_{u \in(0, s)}-\frac{1}{t-s} \rho_{j k}(u) 1_{u \in(s, t)} .
$$

with $\rho$ is the inverse of the volatility matrix $\sigma$. 
Theorem 3.1 For any $s \in(0, t), g \in \mathcal{E}_{b}\left(\mathbb{R}^{d}\right)$ and $x=\left(x_{1}, \ldots, x_{d}\right)$ with $x_{i}>0$,

$$
E\left(g\left(S_{t}\right) \mid S_{s}=x\right)=\frac{T_{s, t}[g](x)}{T_{s, t}[1](x)}
$$

and its partial derivative

$$
\partial_{x_{i}} E\left(g\left(S_{t}\right) \mid S_{s}=x\right)=\frac{R_{s, t}^{i}[g](x) T_{s, t}[1](x)-T_{s, t}[g](x) R_{s, t}^{i}[1](x)}{T_{s, t}[1](x)^{2}},
$$

where $T_{s, t}[f](x)$ and $R_{s, t}^{i}[f](x)$ are defined for every function ${ }^{3} f \in \mathcal{E}_{b}\left(\mathbb{R}^{d}\right)$ by

$$
\begin{gathered}
T_{s, t}[f](x)=E\left(f\left(S_{t}\right) \Gamma_{s, t} \widehat{H}^{x}\left(S_{s}\right)\right) \\
R_{s, t}^{i}[f](x)=-E\left(\frac{f\left(S_{t}\right)}{S_{s}^{i}} \widehat{H}^{x}\left(S_{s}\right)\left(\Gamma_{s, t}\left(1+\pi_{s, t}^{i, d}\right)-\sum_{j=i}^{d} \int_{0}^{t} D_{u}^{j} \pi_{s, t}^{i, d} D_{u}^{j} \Gamma_{s, t} d u\right)\right),
\end{gathered}
$$

with $\widehat{H}^{x}\left(S_{s}\right)=\prod_{k=1}^{d} \frac{H_{k}^{x}\left(S_{s}^{k}\right)}{S_{s}^{k}}, \Gamma_{s, t}$ and $\pi_{s, t}^{k, d}$ are given in Definition 3.1 .

$H_{k}^{x}\left(S_{s}^{k}\right)$ is the Heaviside function of the difference between the $k^{t h}$ stock and the $k^{\text {th }}$ coordinate of the positive vector $x, \mathcal{E}_{b}\left(\mathbb{R}^{d}\right)$ is defined in (16).

Using Theorem 3.1, the conditional expectation in (7) and its derivative are given by (17) and (18). To prove Theorem 3.1, we need the following two lemmas which are proved in [2]. It follows from Lemma 3.1 that the $\operatorname{sum} \sum_{j=i}^{d} \rho_{j i}(u) D_{u}^{j} f\left(S_{t}\right)$ does not depend on $u$.

Lemma 3.1 For any $u \in(0, t), f \in \mathcal{C}^{1}\left(\mathbb{R}^{d}\right)$ and $S$ given by the $S D E(9)$, we have

$$
\sum_{j=i}^{d} \rho_{j i}(u) D_{u}^{j} f\left(S_{t}\right)=S_{t}^{i} \partial_{x_{i}} f\left(S_{t}\right)
$$

The second lemma is based on the duality property of the Malliavin calculus.

Lemma 3.2 For any interval $I \subset(0, t), h \in \mathcal{C}_{b}^{\infty}(\mathbb{R}), F \in \operatorname{Dom}(D)$ and $S$ given by the SDE (9), we have

$$
\begin{aligned}
E\left(\int_{I} \frac{F D_{u}^{i} h\left(S_{s}^{i}\right)}{\sigma_{i i}(u)} d u\right) & =E\left(h\left(S_{s}^{i}\right) F \sum_{j=i}^{d} \int_{I} \rho_{j i}(u) d W_{u}^{j}\right) \\
& -E\left(h\left(S_{s}^{i}\right) \sum_{j=i}^{d} \int_{I} \rho_{j i}(u) D_{u}^{j} F d u\right) .
\end{aligned}
$$

\footnotetext{
${ }^{3}$ In our case $f=g$ or $f=1$
} 
Proof of Theorem 3.1: The equalities (17) and (19) are proved in [2], the new result of this theorem is the partial derivative value (18). Regarding this part, it is sufficient to prove that

$$
\partial_{x_{i}} T_{s, t}[f](x)=R_{s, t}^{i}[f](x) .
$$

Let $\phi \in \mathcal{C}_{c}^{\infty}(\mathbb{R})$ be a mollifier function with support equal to $[-1,1]$ and such that $\int_{\mathbb{R}} \phi(u) d u=1$, then for any $u \in \mathbb{R}$ we define

$$
h_{m i}(u)=\left(H_{i}^{x} * \phi_{m}\right)(u) \in \mathcal{C}_{b}^{\infty}(\mathbb{R}), \quad \phi_{m}(u)=m \phi(m u), \quad m \in \mathbb{N} .
$$

The dependence with respect to $x_{i}$ can be dominated and the differentiation under the integral sign provides

$$
\partial_{x_{i}} E\left(f\left(S_{t}\right) \Gamma_{s, t} \frac{h_{m i}\left(S_{s}^{i}\right)}{S_{s}^{i}} \widehat{H}^{i}\left(S_{s}\right)\right)=-E\left(f\left(S_{t}\right) \Gamma_{s, t} \frac{h_{m i}^{\prime}\left(S_{s}^{i}\right)}{S_{s}^{i}} \widehat{H}^{i}\left(S_{s}\right)\right),
$$

where $\widehat{H}^{i}\left(S_{s}\right)=\prod_{k=1 ; k \neq i}^{d} \frac{H_{k}^{x}\left(S_{s}^{i}\right)}{S_{s}^{i}}$.

Under our assumptions, the distribution of the vector $\left(S_{s}^{1}, \ldots, S_{s}^{d}, S_{t}^{1}, \ldots, S_{t}^{d}\right)$ admits a lognormal joint distribution density $p_{s, t}$ with respect to the Lebesgue measure on $\mathbb{R}_{+}^{d} \times \mathbb{R}_{+}^{d}$. Similar to the argument presented in proof of Theorem 2.1 in [2], using $p_{s, t}$ one gets the limit as $m \longrightarrow \infty$

$$
\partial_{x_{i}} E\left(f\left(S_{t}\right) \Gamma_{s, t} \frac{h_{m i}\left(S_{s}^{i}\right)}{S_{s}^{i}} \widehat{H}^{i}\left(S_{s}\right)\right) \longrightarrow \partial_{x_{i}} T_{s, t}[f](x),
$$

that provides

$$
\partial_{x_{i}} T_{s, t}[f](x)=-\lim _{m \rightarrow+\infty} E\left(f\left(S_{t}\right) \Gamma_{s, t} \frac{h_{m i}^{\prime}\left(S_{s}^{i}\right)}{S_{s}^{i}} \widehat{H}^{i}\left(S_{s}\right)\right) .
$$

We introduce the following notations

$$
\Pi\left(S_{s}\right)=\frac{\widehat{H}^{i}\left(S_{s}\right)}{\left(S_{s}^{i}\right)^{2}}, \quad \widehat{h}_{m i}\left(S_{s}\right)=\frac{h_{m i}\left(S_{s}^{i}\right)}{S_{s}^{i}} \widehat{H}^{i}\left(S_{s}^{i}\right) .
$$

We have by the chain rule $h_{m i}^{\prime}\left(S_{s}^{i}\right)=\frac{D_{u}^{i} h_{m i}\left(S_{s}^{d}\right)}{D_{u}^{i} S_{s}^{i}}$ and $D_{u}^{i} S_{s}^{i}=\sigma_{i i}(u) S_{s}^{i}$ for every $u \in(0, s)$, thus

$$
\begin{aligned}
E\left(f\left(S_{t}\right) \Gamma_{s, t} \frac{h_{m i}^{\prime}\left(S_{s}^{i}\right)}{S_{s}^{i}} \widehat{H}^{i}\left(S_{s}\right)\right) & =E\left(\frac{1}{s} \int_{0}^{s} f\left(S_{t}\right) \Gamma_{s, t} \widehat{H}^{i}\left(S_{s}\right) \frac{D_{u}^{i} h_{m i}\left(S_{s}^{i}\right)}{S_{s}^{i} D_{u}^{i} S_{s}^{i}} d u\right) \\
& =E\left(\frac{1}{s} \int_{0}^{s} f\left(S_{t}\right) \Gamma_{s, t} \widehat{H}^{i}\left(S_{s}\right) \frac{D_{u}^{i} h_{m i}\left(S_{s}^{i}\right)}{\sigma_{i i}(u)\left(S_{s}^{i}\right)^{2}} d u\right) .
\end{aligned}
$$

Using Lemma 3.2 with

$$
F=f\left(S_{t}\right) \Gamma_{s, t} \frac{\widehat{H}^{i}\left(S_{s}\right)}{\left(S_{s}^{i}\right)^{2}}=f\left(S_{t}\right) \Gamma_{s, t} \Pi\left(S_{s}\right),
$$


we get

$$
\begin{aligned}
& E\left(f\left(S_{t}\right) \Gamma_{s, t} \frac{h_{m i}^{\prime}\left(S_{s}^{i}\right)}{S_{s}^{i}} \widehat{H}^{i}\left(S_{s}\right)\right)=E\left(h_{m i}\left(S_{s}^{i}\right) F \frac{1}{s} \sum_{j=i}^{d} \int_{0}^{s} \rho_{j i}(u) d W_{u}^{j}\right) \\
& -E\left(h_{m i}\left(S_{s}^{i}\right) \frac{1}{s} \sum_{j=i}^{d} \int_{0}^{s} \rho_{j i}(u)\left[\begin{array}{r}
\Gamma_{s, t} \Pi\left(S_{s}\right) D_{u}^{j} f\left(S_{t}\right)+f\left(S_{t}\right) \Gamma_{s, t} D_{u}^{j} \Pi\left(S_{s}\right) \\
+f\left(S_{t}\right) \Pi\left(S_{s}\right) D_{u}^{j} \Gamma_{s, t}
\end{array}\right] d u\right) \\
& =E\left(\frac{\widehat{h}_{m i}\left(S_{s}^{i}\right)}{S_{s}^{i}} f\left(S_{t}\right) \frac{1}{s}\left(\Gamma_{s, t}\left(\sum_{j=i}^{d} \int_{0}^{s} \rho_{j i}(u) d W_{u}^{j}+2 s\right)-\sum_{j=i}^{d} \int_{0}^{s} \rho_{j i}(u) D_{u}^{j} \Gamma_{s, t} d u\right)\right) \\
& -E\left(\frac{\widehat{h}_{m i}\left(S_{s}^{i}\right)}{S_{s}^{i}} \Gamma_{s, t} \frac{1}{s}\left(\sum_{j=i}^{d} \int_{0}^{s} \rho_{j i}(u) D_{u}^{j} f\left(S_{t}\right) d u\right)\right)
\end{aligned}
$$

since, using Lemma 3.1, we have

$$
\sum_{j=i}^{d} \rho_{j i}(u) D_{u}^{j} \Pi\left(S_{s}\right)=S_{s}^{i} \partial_{x_{i}} \Pi\left(S_{s}\right)=-2 \Pi\left(S_{s}\right) .
$$

Let us develop the last term in (25), using Lemma 3.1

$$
\begin{aligned}
& E\left(\frac{\widehat{h}_{m i}\left(S_{s}^{i}\right)}{S_{s}^{i}} \Gamma_{s, t} \frac{1}{s}\left(\sum_{j=i}^{d} \int_{0}^{s} \rho_{j i}(u) D_{u}^{j} f\left(S_{t}\right) d u\right)\right) \\
& =E\left(\frac{\widehat{h}_{m i}\left(S_{s}^{i}\right)}{S_{s}^{i}} \frac{1}{t-s}\left(\sum_{j=i}^{d} \Gamma_{s, t} \int_{s}^{t} \rho_{j i}(u) D_{u}^{j} f\left(S_{t}\right) d u\right)\right) \\
& =E\left(\frac{\widehat{h}_{m i}\left(S_{s}^{i}\right)}{S_{s}^{i}} \frac{1}{t-s} \sum_{j=i}^{d} E\left(\Gamma_{s, t} \int_{s}^{t} \rho_{j i}(u) D_{u}^{j} f\left(S_{t}\right) d u \mid \mathcal{F}_{s}\right)\right) \\
& =E\left(\frac{\widehat{h}_{m i}\left(S_{s}^{i}\right)}{S_{s}^{i}} \frac{1}{t-s} \sum_{j=i}^{d} E\left(f\left(S_{t}\right) \Gamma_{s, t} \int_{s}^{t} \rho_{j i}(u) d W_{u}^{j} \mid \mathcal{F}_{s}\right)\right) \\
& =E\left(\frac{\widehat{h}_{m i}\left(S_{s}^{i}\right)}{S_{s}^{i}} \frac{1}{t-s} \sum_{j=i}^{d} E\left(f\left(S_{t}\right)\left(\Gamma_{s, t} \int_{s}^{t} \rho_{j i}(u) d W_{u}^{j}-\int_{s}^{t} \rho_{j i}(u) D_{u}^{j} \Gamma_{s, t} d u\right) \mid \mathcal{F}_{s}\right)\right) .
\end{aligned}
$$

Thus, (25) becomes

$$
\begin{aligned}
& E\left(f\left(S_{t}\right) \Gamma_{s, t} \frac{h_{m i}^{\prime}\left(S_{s}^{i}\right)}{S_{s}^{i}} \widehat{H}^{i}\left(S_{s}\right)\right) \\
& =E\left(\frac{\widehat{h}_{m i}\left(S_{s}^{i}\right)}{S_{s}^{i}} f\left(S_{t}\right)\left(\Gamma_{s, t}\left(1+\pi_{s, t}^{i, d}\right)-\sum_{j=i}^{d} \int_{0}^{t} D_{u}^{j} \pi_{s, t}^{i, d} D_{u}^{j} \Gamma_{s, t} d u\right)\right) .
\end{aligned}
$$

Using a dominated convergence argument, from (24) and (26) we get

$$
\partial_{x_{i}} T_{s, t}[f](x)=-E\left(\frac{f\left(S_{t}\right)}{S_{s}^{i}} \prod_{k=1}^{d} \frac{H_{k}^{x}\left(S_{s}^{k}\right)}{S_{s}^{k}}\left(\Gamma_{s, t}\left(1+\pi_{s, t}^{i, d}\right)-\sum_{j=i}^{d} \int_{0}^{t} D_{u}^{j} \pi_{s, t}^{i, d} D_{u}^{j} \Gamma_{s, t} d u\right)\right) .
$$


Remark $3.1 \quad$ i) Its is important to point out that $\Gamma_{s, t}$ and $\sum_{j=i}^{d} \int_{0}^{t} D_{u}^{j} \pi_{s, t}^{i, d} D_{u}^{j} \Gamma_{s, t} d u$ can be simulated efficiently using the trick given in [2] which will be detailed in Remark 3.2.

ii) For every $g \in \mathcal{E}_{b}\left(\mathbb{R}^{d}\right)$, one can show that the functions $R_{s, t}^{i}[g]$ and $T_{s, t}[g]$ are continuous since the Heaviside function is continuous except on the negligible set $\left\{S_{s}=x\right\}$. The latter fact implies that the partial derivative of the conditional expectation (18) is locally bounded when its payoff is in $\mathcal{E}_{b}\left(\mathbb{R}^{d}\right)$.

\subsection{Backward conditional density estimation}

The authors of [19] proposed a Karhunen-Loeve expansion of Brownian motion and provide in [12] an optimal choice ${ }^{4}$ of the couple (space discretization, probability weights) to approximate the standard Gaussian distribution. Known as quantization, this method of using preloaded files should be the method of choice for problems involving Brownian motion or Brownian bridge. Indeed, for this model, the effectiveness of quantization and its good accuracy for dimensions bigger than 3 make it relevant for various problems. Nevertheless, it is not straightforward to use this method for other multidimensional stochastic volatility or jump diffusion models.

Developed for various models, our method of computing (7) employing Malliavin Calculus is more complex than using directly preloaded files as done with the quantization method. However, one can use also Malliavin Calculus to express the backward conditional density. Provided that we are employing the same pseudo random number generator, one can first approximate the backward conditional density off-line for some points of assets trajectories and store it, then re-use them directly as preloaded files to have the distribution of each contract which is sufficient to compute the CVA or develop any other risk hedging strategy. Moreover, when the backward conditional density is already stored, using it to compute (7) is better, from a variance reduction point of view, than using (17) and (18). In fact, we provide in (29) and (30) the value of the backward conditional density and of its partial derivative. First, let us introduce some notations.

Definition 3.2 The random variables $\bar{\Gamma}_{s, t}$ and $\hat{\Gamma}_{s, t}^{i}$ are the solution $X_{s, t}$ of the following induction scheme

$$
X_{s, t}=X_{s, t}^{1}, X_{s, t}^{k}=X_{s, t}^{k+1} \bar{\pi}_{s, t}^{k, d}-\sum_{j=k}^{d} \int_{0}^{t} D_{u}^{j} X_{s, t}^{k+1} D_{u}^{j} \bar{\pi}_{s, t}^{k, d} d u, \quad k \in\{1, \ldots, d\},
$$

with the terminal values

$$
\bar{\Gamma}_{s, t}^{d+1}=\Gamma_{s, t}, \quad \text { and } \quad \hat{\Gamma}_{s, t}^{d+1, i}=\Gamma_{s, t}\left(1+\pi_{s, t}^{i, d}\right)-\sum_{j=i}^{d} \int_{0}^{t} D_{u}^{j} \pi_{s, t}^{i, d} D_{u}^{j} \Gamma_{s, t} d u,
$$

\footnotetext{
${ }^{4}$ In a sense explained in their paper.
} 
where $\Gamma_{s, t}, \pi_{s, t}^{i, d}$ are defined in Defintion 3.1 and $\bar{\pi}_{s, t}^{k, d}$ is given by $\bar{\pi}_{s, t}^{k, d}=1+\frac{1}{t-s} \sum_{j=k}^{d} \int_{0}^{t} \rho_{j k} d W_{u}^{j}$, with $\rho$ is the inverse of the volatility matrix $\sigma$.

Theorem 3.2 For any $s \in(0, t), g \in \mathcal{E}_{b}\left(\mathbb{R}^{d}\right), x=\left(x_{1}, \ldots, x_{d}\right)$ and $y=\left(y_{1}, \ldots, y_{d}\right)$ with $x_{i}>0$ and $y_{i}>0$,

$$
\begin{aligned}
& T_{s, t}[f](x)=E\left(f\left(S_{t}\right) \bar{h}\left(x, S_{t}\right)\right), \\
& R_{s, t}^{i}[f](x)=E\left(f\left(S_{t}\right) \hat{h}\left(x, S_{t}\right)\right),
\end{aligned}
$$

where

$$
\begin{gathered}
\bar{h}(x, y)=E\left(\Gamma_{s, t} \widehat{H}^{x}\left(S_{s}\right) \mid S_{t}=y\right)=\frac{E\left(\bar{\Gamma}_{s, t} \widehat{H}^{x}\left(S_{s}\right) \widehat{H}^{y}\left(S_{t}\right)\right)}{E\left(\Gamma_{s, t} \widehat{H}^{y}\left(S_{t}\right)\right)} \\
\hat{h}(x, y)=E\left(\left(\Gamma_{s, t}\left(1+\pi_{s, t}^{i, d}\right)-\sum_{j=i}^{d} \int_{0}^{t} D_{u}^{j} \pi_{s, t}^{i, d} D_{u}^{j} \Gamma_{s, t} d u\right) \frac{\widehat{H}^{x}\left(S_{s}\right)}{S_{s}^{i}} \mid S_{t}=y\right) \\
=\frac{E\left(\frac{\hat{\Gamma}_{s, t}}{S_{s}^{i}} \widehat{H}^{x}\left(S_{s}\right) \widehat{H}^{y}\left(S_{t}\right)\right)}{E\left(\Gamma_{s, t} \widehat{H}^{y}\left(S_{t}\right)\right)},
\end{gathered}
$$

with $\widehat{H}^{y}\left(S_{t}\right)=\prod_{j=1}^{d} \frac{H_{j}^{y}\left(S_{t}^{j}\right)}{S_{t}^{j}}, \Gamma_{s, t}, \pi_{s, t}^{i, d}, \bar{\Gamma}_{s, t}$ and $\hat{\Gamma}_{s, t}$ are given in Definition 3.1 and Definition 3.2.

Proof of Theorem 3.2: We will prove that for $h_{i} \in \mathcal{C}_{b}^{\infty}(\mathbb{R})$ and $0 \leq k \leq d$

$$
E\left(\Gamma_{s, t} \widehat{H}^{x}\left(S_{s}\right) \prod_{i=1}^{d} h_{i}^{\prime}\left(S_{t}^{i}\right)\right)=E\left(\bar{\Gamma}_{s, t}^{k+1} \widehat{H}^{x}\left(S_{s}\right) \prod_{i=1}^{k} h_{i}^{\prime}\left(S_{t}^{k}\right) \prod_{i=k+1}^{d} \frac{h_{i}\left(S_{t}^{k}\right)}{S_{t}^{k}}\right),
$$

with (27) obtained directly from (31) when $k=0$. In fact, $E\left(\Gamma_{s, t} \prod_{j=1}^{d} \frac{H_{j}\left(S_{s}^{j}\right)}{S_{s}^{j}} \mid S_{t}=y\right)$ can be viewed heuristically as $\frac{E\left(\Gamma_{s, t} \prod_{j=1}^{d} \frac{H_{j}\left(S_{s}^{j}\right)}{S_{s}^{j}} \varepsilon_{y}\left(S_{t}\right)\right)}{E\left(\varepsilon_{y}\left(S_{t}\right)\right)}$ where $\varepsilon_{y}$ is the Dirac distribution at $y$ and we know that $\varepsilon_{y_{i}}=H_{i}^{\prime}$. In order to make the reasoning rigorous, one replace the expectation of $\varepsilon_{y}\left(S_{t}\right)$ by the density of $S_{t}$ evaluated at $y$.

Now let us prove (31) by induction, we introduce the following notations

$$
\widehat{h}_{k}^{d}\left(S_{t}\right)=\prod_{i=k}^{d} \frac{h_{i}\left(S_{t}^{i}\right)}{S_{t}^{i}}, \quad{\widehat{h^{\prime}}}_{k}\left(S_{t}\right)=\prod_{i=1}^{k} h_{i}^{\prime}\left(S_{t}^{i}\right) .
$$


When $k=d$, we have by the chain rule $h_{d}^{\prime}\left(S_{t}^{d}\right)=\frac{D_{u}^{d} h_{d}\left(S_{t}^{d}\right)}{D_{u}^{d} S_{t}^{d}}$ and $D_{u}^{d} S_{t}^{d}=\sigma_{d d}(u) S_{t}^{d}$ for every $u \in(s, t)$, thus

$$
\begin{aligned}
E\left(\Gamma_{s, t} \widehat{H}^{x}\left(S_{s}\right){\widehat{h^{\prime}}}_{d}\left(S_{t}\right)\right) & =E\left(\frac{1}{t-s} \int_{s}^{t} \Gamma_{s, t} \widehat{H}^{x}\left(S_{s}\right){\widehat{h^{\prime}}}_{d-1}\left(S_{t}\right) \frac{D_{u}^{d} h_{d}\left(S_{t}^{d}\right)}{D_{u}^{d} S_{t}^{d}} d u\right) \\
& =E\left(\frac{1}{t-s} \int_{s}^{t} \Gamma_{s, t} \widehat{H}^{x}\left(S_{s}\right) \widehat{h}^{\prime}{ }_{d-1}\left(S_{t}\right) \frac{D_{u}^{d} h_{d}\left(S_{t}^{d}\right)}{\sigma_{d d}(u) S_{t}^{d}} d u\right) .
\end{aligned}
$$

Using Lemma 3.2 with

$$
F=\frac{\Gamma_{s, t}}{S_{t}^{d}} \widehat{H}^{x}\left(S_{s}\right){\widehat{h^{\prime}}}_{d-1}\left(S_{t}\right)
$$

and the fact that ${\widehat{h^{\prime}}}_{d-1}\left(S_{s}\right)$ does not depend on the $d^{\text {th }}$ coordinate of the Brownian motion yields

$$
\begin{aligned}
& E\left(\Gamma_{s, t} \widehat{H}^{x}\left(S_{s}\right) \widehat{h}_{d}^{\prime}\left(S_{t}\right)\right) \\
& =E\left(F h_{d}\left(S_{t}^{d}\right) \frac{1}{t-s} \int_{s}^{t} \frac{d W_{u}^{d}}{\sigma_{d d}(u)}\right)-E\left(h_{d}\left(S_{t}^{d}\right) \frac{1}{t-s} \int_{s}^{t} D_{u}^{d} F \frac{d u}{\sigma_{d d}(u)}\right) \\
& =E\left(h_{d}\left(S_{t}^{d}\right) \widehat{H}^{x}\left(S_{s}\right) \widehat{h}_{d-1}^{\prime}\left(S_{t}\right)\left(\frac{\Gamma_{s, t}}{S_{t}^{d}}\left(\bar{\pi}_{s, t}^{d, d}-1\right)-\frac{1}{t-s} \int_{s}^{t} D_{u}^{d} \frac{\Gamma_{s, t}}{S_{t}^{d}} \frac{d u}{\sigma_{d d}(u)}\right)\right) \\
& =E\left(\frac{h_{d}\left(S_{t}^{d}\right)}{S_{t}^{d}} \widehat{H}^{x}\left(S_{s}\right){\widehat{h^{\prime}}}_{d-1}\left(S_{t}\right)\left(\Gamma_{s, t} \bar{\pi}_{s, t}^{d, d}-\frac{1}{t-s} \int_{s}^{t} D_{u}^{d} \Gamma_{s, t} \frac{d u}{\sigma_{d d}(u)}\right)\right) \\
& =E\left(\bar{\Gamma}_{s, t}^{d} \widehat{H}^{x}\left(S_{s}\right) \widehat{h}_{d-1}^{\prime}\left(S_{t}\right) \frac{h_{d}\left(S_{t}^{d}\right)}{S_{t}^{d}}\right)
\end{aligned}
$$

where the equality (32) comes from the fact that for $u \in(s, t)$

$$
\frac{1}{\sigma_{d d}} D_{u}^{d} \frac{\Gamma_{s, t}}{S_{t}^{d}}=\frac{1}{S_{t}^{d} \sigma_{d d}} D_{u}^{d} \Gamma_{s, t}-\frac{\Gamma_{s, t}}{S_{t}^{d}}
$$

Now, let us suppose that (31) is satisfied for $k$ and prove it for $k-1$. We have by the chain rule $h_{k}^{\prime}\left(S_{s}^{k}\right)=\frac{D_{u}^{k} h_{k}\left(S_{s}^{k}\right)}{D_{u}^{k} S_{s}^{k}}$ and $D_{u}^{k} S_{s}^{k}=\sigma_{k k}(u) S_{s}^{k}$, thus

$$
\begin{aligned}
E\left(\Gamma_{s, t} \widehat{H}^{x}\left(S_{s}\right) \widehat{h}_{d}^{\prime}\left(S_{t}\right)\right) & =E\left(\bar{\Gamma}_{s, t}^{k+1} \widehat{H}^{x}\left(S_{s}\right){\widehat{h^{\prime}}}_{k}\left(S_{t}\right) \widehat{h}_{k+1}^{d}\left(S_{t}\right)\right) \\
& =E\left(\frac{1}{t-s} \int_{s}^{t} \bar{\Gamma}_{s, t}^{k+1} \widehat{H}^{x}\left(S_{s}\right) \widehat{h}_{k-1}^{\prime}\left(S_{t}\right) \widehat{h}_{k+1}\left(S_{t}\right) \frac{D_{u}^{k} h_{k}\left(S_{t}^{k}\right)}{\sigma_{k k}(u) S_{t}^{k}} d u\right) .
\end{aligned}
$$

As before, using Lemma 3.2 with in this time

$$
F=\frac{\bar{\Gamma}_{s, t}^{k+1}}{S_{t}^{k}} \widehat{H}^{x}\left(S_{s}\right) \widehat{h}_{k-1}^{\prime}\left(S_{t}\right) \widehat{h}_{k+1}\left(S_{t}\right)
$$


and the fact that ${\widehat{h^{\prime}}}_{k-1}\left(S_{t}\right)$ and $\widehat{h}_{k+1}\left(S_{t}\right)$ are not depend on the $k^{t h}$ coordinate of the Brownian motion, we obtain

$$
\begin{aligned}
& E\left(\Gamma_{s, t} \widehat{H}^{x}\left(S_{s}\right) \widehat{h}_{d}^{\prime}\left(S_{t}\right)\right) \\
& =\sum_{i=k}^{d} E\left(h_{k}\left(S_{s}^{k}\right) \widehat{h}_{k+1}\left(S_{t}\right){\widehat{h^{\prime}}}_{k-1}\left(S_{t}\right) \frac{\widehat{H}^{x}\left(S_{s}\right)}{t-s}\left(\frac{\bar{\Gamma}_{s, t}^{k+1}}{S_{t}^{k}} \int_{s}^{t} \rho_{i k}(u) d W_{u}^{i}-\int_{s}^{t} \rho_{i k}(u) D_{u}^{i} \frac{\bar{\Gamma}_{s, t}^{k+1}}{S_{t}^{k}} d u\right)\right) \\
& =E\left(\widehat{H}^{x}\left(S_{s}\right){\widehat{h^{\prime}}}_{k-1}\left(S_{t}\right) \widehat{h}_{k}\left(S_{t}\right)\left(\bar{\Gamma}_{s, t}^{k+1} \bar{\pi}_{s, t}^{k, d}-\frac{1}{t-s} \int_{s}^{t} D_{u}^{i} \bar{\Gamma}_{s, t}^{k+1} D_{u}^{i} \bar{\pi}_{s, t}^{k, d} d u\right)\right) \\
& =E\left(\bar{\Gamma}_{s, t}^{k} \widehat{H}^{x}\left(S_{s}\right) \widehat{h}_{k-1}^{\prime}\left(S_{t}\right) \widehat{h}_{k}\left(S_{t}\right)\right)
\end{aligned}
$$

Similarly, one can prove (28).

In Remark 3.2, we use the set of the second order permutations $\overline{\mathcal{P}}_{1, d}$ defined by

$$
\overline{\mathcal{P}}_{1, d}=\left\{p \in \mathcal{P}_{1, d} ; p \circ p=I d\right\},
$$

where $\mathcal{P}_{1, d}$ is the set of permutations on $\{1, \ldots, d\}$ and $I d$ is the identity application.

Remark 3.2 In order to make easier the implementation of (19), it was shown in [2] that $\Gamma_{s, t}$ given in Definition 3.1 can be computed as a determinant of a given matrix $A$

$$
\Gamma_{s, t}=\sum_{p \in \overline{\mathcal{P}}_{1, d}} \epsilon(p) \prod_{i=1}^{d} A_{i, p(i)}
$$

with $\epsilon(p)$ as the signature of the permutation $p \in \overline{\mathcal{P}}_{1, d}$, and

$$
A=\left(\begin{array}{ccccc}
\pi_{s, t}^{1, d} & C_{1,2} & C_{1,3} & \ldots & C_{1, d} \\
1 & \pi_{s, t}^{2, d} & C_{2,3} & \ldots & C_{2, d} \\
\vdots & \ddots & \ddots & \ddots & \vdots \\
1 & \ldots & 1 & \pi_{s, t}^{d-1, d} & C_{d-1, d} \\
1 & 1 & \ldots & 1 & \pi_{s, t}^{d, d}
\end{array}\right)
$$

where

$$
C_{k, l}=\operatorname{Cov}\left(\pi_{s, t}^{k, d}, \pi_{s, t}^{l, d}\right)=\sum_{j=k \vee l}^{d}\left(\frac{1}{s^{2}} \int_{0}^{s} \rho_{j k}(u) \rho_{j l}(u) d u+\frac{1}{(t-s)^{2}} \int_{s}^{t} \rho_{j k}(u) \rho_{j l}(u) d u\right) .
$$

Using the same idea, we can deduce a generating method for the computation of $\bar{\Gamma}_{s, t}$ and $\hat{\Gamma}_{s, t}$. In fact, the solution $X_{s, t}$ of the induction scheme in Definition 3.2 can be expressed in a similar way as follow

$$
X_{s, t}=\sum_{p \in \overline{\mathcal{P}}_{1, d+1}} \epsilon(p) \prod_{i=1}^{d+1} \bar{A}_{i, p(i)}
$$


with $\epsilon(p)$ is the signature of the permutation $p \in \overline{\mathcal{P}}_{1, d+1}$ and

$$
\bar{A}=\left(\begin{array}{ccccc}
\bar{\pi}_{s, t}^{1, d} & \bar{C}_{1,2} & \bar{C}_{1,3} & \cdots & \bar{C}_{1, d+1} \\
1 & \bar{\pi}_{s, t}^{2, d} & \bar{C}_{2,3} & \cdots & \bar{C}_{2, d+1} \\
\vdots & \ddots & \ddots & \ddots & \vdots \\
1 & \cdots & 1 & \bar{\pi}_{s, t}^{d, d} & \bar{C}_{d, d+1} \\
1 & 1 & \cdots & 1 & X_{s, t}^{d+1, d}
\end{array}\right)
$$

where $\bar{C}_{k, d+1}=\left\langle\bar{\pi}_{s, .}^{k, d}, X_{s, .}^{d+1, d}\right\rangle_{t}$ and

$$
\bar{C}_{k, l}=\operatorname{Cov}\left(\bar{\pi}_{s, t}^{k, d}, \bar{\pi}_{s, t}^{l, d}\right)=\sum_{j=k \vee l}^{d} \frac{1}{(t-s)^{2}} \int_{s}^{t} \rho_{j k}(u) \rho_{j l}(u) d u, k, l \in\{1, . ., d\} .
$$

Let denote $\Gamma_{s, t}^{-k}$ the second order permutation determinant of the matrix $A^{-k}$ that comes from $A$ by suppressing both line and column "k"

$$
\Gamma_{s, t}^{-k}=\sum_{p \in \overline{\mathcal{P}}_{1, d}^{k}} \epsilon(p) \prod_{i=1 ; i \neq k}^{d} A_{i, p(i)}
$$

where $\overline{\mathcal{P}}_{1, d}^{k}=\left\{p \in \overline{\mathcal{P}}_{1, d}, \quad p(k)=k\right\}$. The following lemma gives a generating way to implement the term " $\sum_{j=i}^{d} \int_{0}^{t} D_{u}^{j} \pi_{s, t}^{i, d} D_{u}^{j} \Gamma_{s, t} d u$ " which appear in both Theorem 3.1 and Theorem 3.2.

Lemma 3.3 Let $i \in\{1, \ldots, d\}$ and $s \in(0, t)$. We have

$$
\sum_{j=i}^{d} \int_{0}^{t} D_{u}^{j} \pi_{s, t}^{i, d} D_{u}^{j} \Gamma_{s, t} d u=\sum_{l=1}^{d} \Gamma_{s, t}^{-l} C_{l, i}
$$

Proof of Lemma 3.3: First, using (34), we have for every $u \in(0, t)$

$$
\begin{aligned}
D_{u}^{j} \Gamma_{s, t} & =\sum_{p \in \overline{\mathcal{P}}_{1, d}} \epsilon(p) D_{u}^{j}\left(\prod_{i=1}^{d} A_{i, p(i)}\right) \\
& =\sum_{p \in \overline{\mathcal{P}}_{1, d}} \epsilon(p) \sum_{l=1}^{d}\left(\prod_{i=1 ; i \neq l}^{d} A_{i, p(i)}\right) D_{u}^{j} A_{l, p(l)} \\
& =\sum_{l=1}^{d} \sum_{p \in \overline{\mathcal{P}}_{1, d}^{l}} \epsilon(p)\left(\prod_{i=1 ; i \neq l}^{d} A_{i, p(i)}\right) D_{u}^{j} \pi_{s, t}^{l, d}
\end{aligned}
$$

where the last equality is due to the fact that $A_{l, p(l)}$ is deterministic except for $p(l)=l$ for which $A_{l, l}=\pi_{s, t}^{l, d}$. Now, since $\pi_{s, t}^{l, d}$ does not depend on the Brownien 
motion $W^{j}$ when $l>j$, using Fubini theorem we get

$$
\begin{aligned}
\sum_{j=i}^{d} \int_{0}^{t} D_{u}^{j} \pi_{s, t}^{i, d} D_{u}^{j} \Gamma_{s, t} d u & =\sum_{j=i}^{d} \sum_{l=1}^{j} \sum_{p \in \overline{\mathcal{P}}_{1, d}^{l}} \epsilon(p) \prod_{i=1 ; i \neq l}^{d} A_{i, p(i)} \int_{0}^{t} D_{u}^{j} \pi_{s, t}^{i, d} D_{u}^{j} \pi_{s, t}^{l, d} d u \\
& =\sum_{l=1}^{d} \sum_{j=l \vee i}^{d} \sum_{p \in \overline{\mathcal{P}}_{1, d}^{l}} \epsilon(p) \prod_{i=1 ; i \neq l}^{d} A_{i, p(i)} \int_{0}^{t} D_{u}^{j} \pi_{s, t}^{i, d} D_{u}^{j} \pi_{s, t}^{l, d} d u \\
& =\sum_{l=1}^{d} \Gamma_{s, t}^{-l} C_{l, i} .
\end{aligned}
$$

\section{Complexity comparison}

\subsection{Square Monte Carlo benchmark \& regression method}

As we saw in the introduction, to approximate the CVA value using (6), one needs first to simulate assets trajectories, also known as simulating scenarios [10], that corresponds to the outer expectation, then compute the conditional expectation given by (7) involved in (3), (4) and (5). For some models and some contracts, this computation could be explicit or semi-explicit using Fourier transform [8]. When the dimension is 2 or 3 and for a large choice of models and contracts, numerical methods for $\mathrm{PDEs}^{5}$ become more appropriate because faster than a Monte Carlo simulation. However, for the most general models and contracts and for dimensions bigger than 4, the only option that remains is the use of Monte Carlo for both simulating asset trajectories and the conditional expectation in (7).

When only exchanging European contracts with the counterparty and the number of simulated trajectories is big enough, using a square Monte Carlo for CVA computation gives reference results. The parallel suitability of Monte Carlo allows to increase the accuracy using more computing resources including many-cores architectures as done in [1]. Nevertheless, the complexity of square Monte Carlo remains too high. Indeed, if we denote by $d$ the dimension of the problem in terms of assets, by $M$ the number of simulated trajectories and by $N$ the number of time steps of the simulation $^{6}$, then we shall simulate exactly $d N M[1+(M-1)(N-1) / 2]$ segments of trajectory (see Figure 1). The proof of this result is built on the assumption that we use the same number of trajectories for both Monte Carlo simulations involved. In fact, we have to draw $d M N$ segments for the first Monte Carlo and at each time step $t_{k}$, we should simulate $(M-1)(N-k)$ segments of trajectories at each point

\footnotetext{
${ }^{5}$ Partial Differential Equations

${ }^{6}$ Supposed here equal to the number of time steps of the SDE discretization
} 


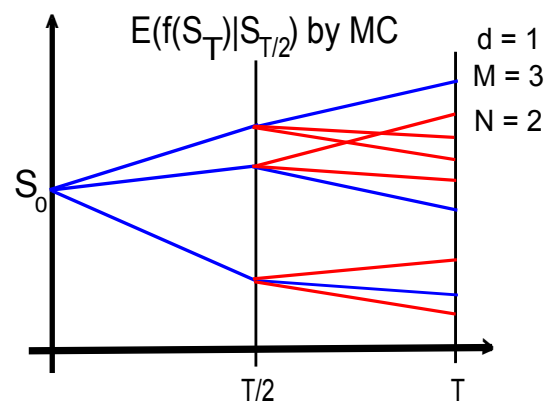

Figure 1: The price of the contract at $T / 2$ is approximated by a Monte Carlo simulation.

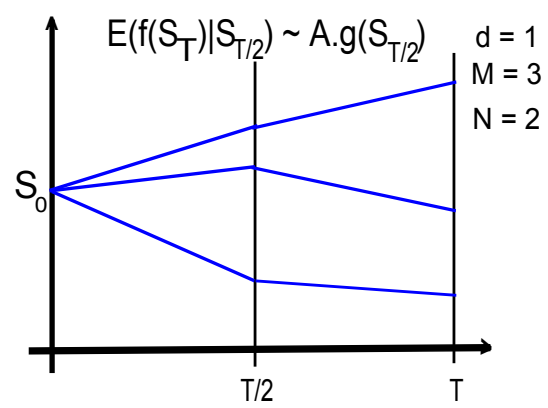

Figure 2: The price of the contract at $T / 2$ is approximated by a linear regression on a finite basis.

from the first $d M N$ set, which leads to

$$
d M N+\sum_{k=1}^{N} d M(M-1)(N-k)=d M N\left(1+\frac{(M-1)(N-1)}{2}\right) .
$$

Thus, if we denote by $\mathcal{T}$ the complexity of the simulation of a segment of a trajectory, the complexity of square Monte Carlo is of order $O\left(\mathcal{T} d M^{2} N^{2}\right)$. Another inconvenient of this method comes from the fact that it has an exponential complexity according to the number of trajectories when employed for American contracts. Indeed, according to induction (5), to get $\phi_{t_{k}}^{a m}\left(S_{t_{k}}\right)$ we must have $M$ trajectories of the function $\phi_{t_{k+1}}^{a m}\left(S_{t_{k+1}}\right)$ and so on.

For all the previous reasons, practitioners prefer the use of methods based on regression whose complexity can be decomposed into two terms: A part associated to the scenarios generation which is of order $O(\mathcal{T} d M N)$ and another part for the approximation of the conditional expectation by a regression on a polynomial basis $b\left(S_{t_{k}}\right): E\left(f\left(S_{t_{k+1}}\right) \mid S_{t_{k}}\right) \approx A . b\left(S_{t_{k}}\right)$ (see Figure 2). The vector $A$ minimizes the quadratic error

$$
\left\|f\left(S_{t_{k+1}}\right)-A . b\left(S_{t_{k}}\right)\right\|_{L^{2}}
$$

and thus equal to

$$
A=\Psi^{-1} E\left(f\left(S_{t_{k+1}}\right) b\left(S_{t_{k}}\right)\right),
$$

where the matrix $\Psi=E\left(b\left(S_{t_{k}}\right) b^{t}\left(S_{t_{k}}\right)\right)$. Consequently, at each time step, the matrix inversion (36) can be implemented by the Singular Value Decomposition (SVD) explained in [18] and the expectations are approximated by an arithmetic average

$$
E\left(f\left(S_{t_{k+1}}\right) b\left(S_{t_{k}}\right)\right) \approx \frac{1}{N} \sum_{l=1}^{N} f\left(S_{t_{k+1}}^{(l)}\right) b\left(S_{t_{k}}^{(l)}\right)
$$




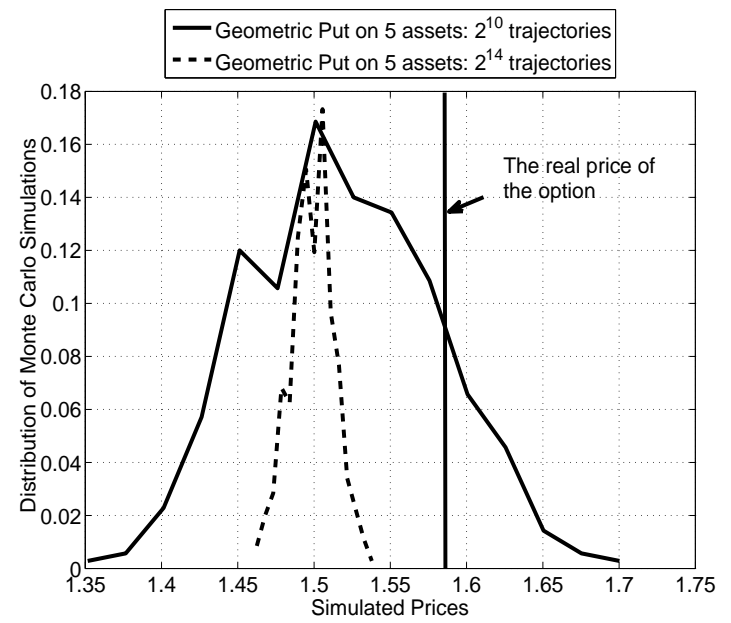

Figure 3: Histogram of prices obtained by Monte Carlo using Longstaff-Schwartz algorithm with maturity $T=1$ and thirty exercise dates.

$$
E\left(b\left(S_{t_{k}}\right) b^{t}\left(S_{t_{k}}\right)\right) \approx \frac{1}{N} \sum_{l=1}^{N} b\left(S_{t_{k}}^{(l)}\right) b^{t}\left(S_{t_{k}}^{(l)}\right) .
$$

Due to the SVD inversion performed at each time step after an expectation approximation, the complexity of the regression phase is of order $O\left(K_{M}^{3} M N\right)$, where $K_{M}$ is the cardinal of the vector $b\left(S_{t_{k}}\right)$. The overall regression method for CVA computation has a complexity of order $O\left(\left(\mathcal{T} d+K_{M}^{3}\right) M N\right)$ even when American contracts are involved. Although this method is less complex than square Monte Carlo, it is not well suited to parallel implementation (see [1]) because of the matrix inversion phase. Moreover, the accuracy cannot be increased only by increasing the number of trajectories, because one has also to take bigger values of $K_{M}$. The latter fact becomes a real issue when the dimension $d$ is big enough which could be seen in Figure 3 for $d=5$, as the obtained prices remain far from the real price even when taking more trajectories. This limitation was studied by the authors of [17] that recommend to have a number of polynomials $K_{M} \sim O(\sqrt{\log (M)})$. Despite of these drawbacks and because this method is quite fast, practitioners, like in [10], prefer to use it with some heuristics to improve the simulated results.

\subsection{MCM as an alternative}

From a theoretical point of view, MCM is really interesting because it is general enough to a large choice of models and contracts. Thus, unlike regression methods, there is no heuristic choice of the vector $g\left(S_{t_{k}}\right)$ that should be specified according to the model and to the contract. Indeed, as already mentioned in Section 2 as well as in [2] and will be discussed also in Section 5, we can apply MCM to pathdependent contracts and with models as multidimensional Heston models and usual jump diffusion models and others. From a computational point of view, because it 


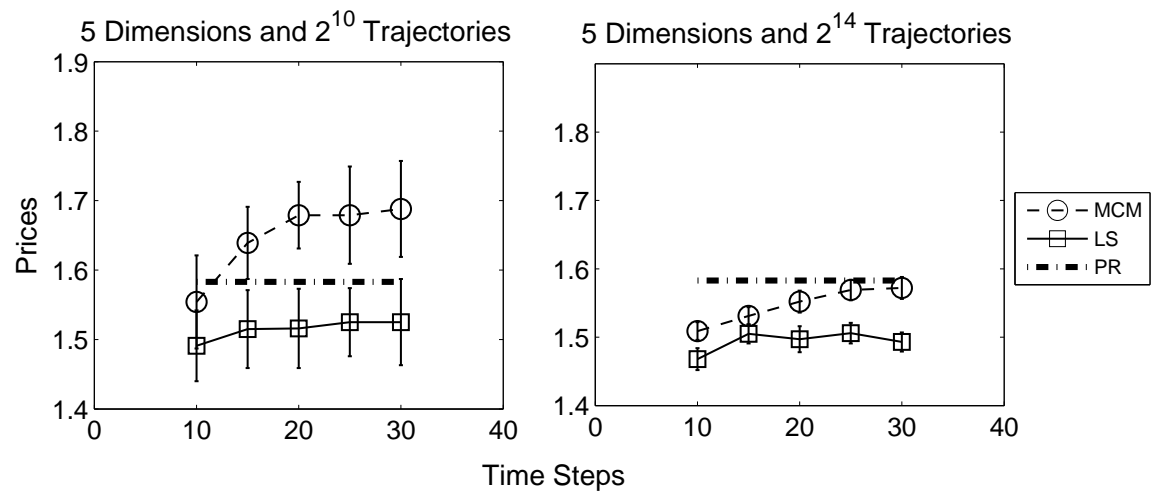

Figure 4: LS vs. MCM for 5 dimensional American option, the real price is given by PR.

is a pure Monte Carlo method, MCM is as suited to parallel implementation as a square Monte Carlo method. MCM could be also used for CVA computation that involves American contracts. Also, in contrast to regression algorithms, MCM aims at a nonparametric algorithm in which the accuracy can be improved by increasing only the number of the simulated trajectories and adding more computing resources to speed up simulations. This fact is demonstrated on Figure 4 in which we can see that MCM performs better ${ }^{7}$ than Longstaff-Schwartz (LS) algorithm ${ }^{8}$ when we increase the number of simulated trajectories. Moreover, according to Figure 4, MCM is as efficient as LS because it could provide sufficiently good results even when simulating very few number of trajectories, here only $2^{10}$. In Section 5 , we will see that the accurate results obtained by MCM goes beyond the computation of the CVA, because it allows to have compelling approximation of the delta sensitivity.

Although MCM is both less complex than square Monte Carlo and applicable for CVA involving American contracts, it is more complex than regression methods. Indeed, with MCM we need only to simulate the assets trajectories once, but the approximation of the conditional expectation as a quotient of two expectations is implemented for each path. The complexity of this method is then of order $O\left(C_{d} \mathcal{T} d M^{2} N\right)$, where $C_{d}$ depends on the dimension $d$. If the backward conditional density, introduced in Section 3, is computed off-line then $C_{d}=1$. Otherwise, $C_{d}=O\left(n_{d}\right)$ where $n_{d}$ is the number of terms involved in the quasi-determinant $\Gamma_{s, t}$ and expressed in the following proposition.

Proposition 4.1 For $d \geq 3, n_{d}$ satisfies the following induction

$$
n_{d}=n_{d-1}+(d-1) n_{d-2}
$$

with $n_{1}=1, n_{2}=2$.

Proposition 4.1 can be easily proved by induction using the relation

$$
\overline{\mathcal{P}}_{1, d}=\left\{\tau_{d-1}^{d-1} \circ p ; p \in \overline{\mathcal{P}}_{1, d-1}\right\} \cup\left\{\tau_{d-1}^{l} \circ p ; p \in \overline{\mathcal{P}}_{1, d-1}, p(l)=l, l \in\{1, \ldots, d-1\}\right\},
$$

\footnotetext{
${ }^{7}$ When only American options are involved. The error should be bigger when simulating the CVA on various American options

${ }^{8}$ An algorithm based on regression.
} 
where $\tau_{i}^{j}: i \leftrightarrow j$ is the transposition application on $\{1, \ldots, d-1\}$ that swaps only $i$ to $j$ and $j$ to $i$. This relation is another version of the relation (2.16) given in [2]. Although the induction (37) provides an $n_{d}$ which is by far smaller than $d$ !, $n_{d}$ can be quite big for high dimensions, for example: $n_{5}=26, n_{6}=76, n_{7}=232$ and $n_{10}=9496$. To overcome this problem, we explain, in the example given in Section 5 how to group contracts together in order to reduce the number of Brownian motions involved and reduce $d$.

\section{Numerical tests using parallel implementa- tion}

After presenting the theoretical framework of CVA estimation using MCM and studying its computational complexity, we demonstrate here the accuracy of this method by comparing it to square Monte Carlo and to a regression method. When using large number of trajectories, square Monte Carlo provides benchmark values for both path-independent and path-dependent European contracts that will be considered as the real values. Consequently, we study the accuracy of MCM for CVA that involves only European contracts. In order to have an idea of how MCM behaves when dealing with American contracts, we refer the reader to [2]. Before summarizing the results of the different simulations, we start by presenting the considered models and how simulating European path-dependent contracts could be performed.

\subsection{Benchmarking setup}

Based on Section 2, we implement one example from the intensity family and one example from the structural family. Each model will be completely specified by the assets dynamic and either the dependence between the assets $S_{t}$ and the default time $\tau$ or between the value exposure $V_{t}$ and $\tau$.

Regarding the intensity model, we take the constant volatility version of (9):

$$
\left\{\begin{array}{c}
\frac{d S_{t}^{i}}{S_{t}^{i}}=r d t+\sigma_{i} \sum_{j=1}^{i} \varrho_{i j} d W_{t}^{j}, \quad S_{0}^{i}=z_{i}, \quad i=1, . ., d, \\
\lambda_{t}=\alpha^{\prime} V_{t}
\end{array}\right.
$$

with $T=1$, the risk neutral interest rate $r=\ln (1.1)$, the time discretization is defined using the time steps that is given as a parameter in each simulation, $S_{0}^{i}=100$ and $\sigma_{i j}=\sigma_{i} \varrho_{i j}$ with $\sigma_{i}=0.2$ where $\varrho=\left\{\varrho_{i j}\right\}_{1 \leq i, j \leq d}$ comes from the Cholesky decomposition of the correlation matrix $\delta_{i-j}+\alpha\left(1-\delta_{i-j}\right)$ such that $\alpha \in[0,1)$ and $\delta$ is the Kronecker symbol. Finally, $\lambda_{t}$ given in (12) is assumed to be equal to $\lambda_{t}=\alpha^{\prime} V_{t}$ with $\alpha^{\prime}>0$.

Regarding the structural model, we take the constant volatility version of (9) and we correlate it with the Brownian motion $W_{t}^{0}$ that drives the firm value process 
$X_{t}$ :

$$
\left\{\begin{array}{c}
\frac{d X_{t}}{X_{t}}=r d t+\sigma_{0} d W_{t}^{0}, \quad X_{0}=z_{0} \\
\frac{d S_{t}^{i}}{S_{t}^{i}}=r d t+\sigma_{i} \sum_{j=0}^{i} \varrho_{i j} d W_{t}^{j}, \quad S_{0}^{i}=z_{i}, \quad i=1, . ., d
\end{array}\right.
$$

with $T=1$, the risk neutral interest rate $r=\ln (1.1)$, the time discretization is defined using the time steps that is given as a parameter in each simulation, $S_{0}^{i}=100$ and $\sigma_{i j}=\sigma_{i} \varrho_{i j}$ with $\sigma_{i}=0.2$ where $\varrho=\left\{\varrho_{i j}\right\}_{0 \leq i, j \leq d}$ comes from the Cholesky decomposition of the correlation matrix $\delta_{i-j}+\alpha\left(1-\delta_{i-j}\right)$ such that $\alpha \in[0,1)$ and $\delta$ is the Kronecker symbol. With this specific example, one understands better the sense of (15) and how the Malliavin Calculus can be implemented on $W=\left(W^{1}, \ldots, W^{d}\right)$ without considering the dependence with respect to $W^{0}$.

Using MCM, simulating CVA that involves only European path-independent contracts (3) is quite simple when compared to adding European path-dependent contracts (4). Then, some details should be provided for the implementation of (4). To simplify the explanations, we assume that $N$ involved in (6) is equal to the number of time steps used to approximate the trajectories of the assets. Let us consider the problem of approximating the conditional expectation

$$
E\left(\left(\max _{i=0, . ., N} S_{t_{i}}^{1}-S_{t_{N}}^{2}\right)_{+} \mid \mathcal{F}_{t_{k}}\right)
$$

Using Markov property and notation $\bar{S}_{t_{k}}^{1}=\max _{i=0, . ., k} S_{t_{i}}^{1}$, this conditional expectation can be rewritten as

$$
\begin{aligned}
& E\left(\left(\max _{i=0, . ., N} S_{t_{i}}^{1}-S_{t_{N}}^{2}\right)_{+} \mid \mathcal{F}_{t_{k}}\right)=E\left(\left(\bar{S}_{t_{k}}^{1} \vee_{i=k+1, . ., N} \max _{t_{i}}^{1}-S_{t_{N}}^{2}\right)_{+} \mid \bar{S}_{t_{k}}^{1}, S_{t_{k}}^{1}, S_{t_{k}}^{2}\right) \\
& =\quad \Theta_{S_{t_{k}}^{1}, S_{t_{k}}^{2}}\left(\bar{S}_{t_{k}}^{1}\right)
\end{aligned}
$$

with:

$$
\Theta_{S_{t_{k}}^{1}, S_{t_{k}}^{2}}(y)=E\left(\left(y \vee \max _{i=k+1, . ., N} S_{t_{i}}^{1}-S_{t_{N}}^{2}\right)_{+} \mid S_{t_{k}}^{1}, S_{t_{k}}^{2}\right)
$$

and for each asset trajectory, we have a specific value $y$ and once it is fixed as a payoff parameter, we can compute the conditional expectation using the result of Theorem 3.1 or Theorem 3.2.

The previous idea for implementing European path-dependent contract can be applied for lookback as well as barrier and Asian contract. In order to have a better approximation of some SDEs and path-dependent contracts, one can also take the number of time steps bigger than $N^{9}$. Indeed, considering the previous path-dependent example, it is possible to increase the accuracy of the approximation

\footnotetext{
${ }^{9}$ We remind the reader that $N$ is associated to the discretization of the time integral.
} 
of $\sup _{0 \leq s \leq t_{k}} S_{s}$ and $\sup _{t_{k}<s \leq t_{N}} S_{s}$ if we have more than $k$ points and $N-k$ points in the intervals $\left[0, t_{k}\right]$ and $\left[t_{k}, t_{N}\right]$ respectively.

We should also discuss the parameters $C_{d}$ and $n_{d}$ introduced in Section 4.2. Previously, we pointed out that the induction (37) provides important values when $d$ is big. However, not all contracts require the d-dimensional information, fact that can be seen with our previous example given in (41). Actually, assuming $d=10$, to evaluate (40) we need only two Brownian motions if we are using intensity model (38) and three Brownian motions ${ }^{10}$ when using structural model (39).

We finish the benchmarking setup by a remark related to the Monte Carlo estimator of (19) and (20). In order to approximate numerically the previous values, we remind that we are using the same set of trajectories which makes MCM less complex than a square Monte Carlo. However, some trajectories must not be added ${ }^{11}$ to the Monte Carlo estimator of $T_{s, t}[f](x)$ and $R_{s, t}^{i}[f](x)$ because they are far from the point $x$. This technique is known by localization and some papers, as [3, 20], propose some "optimal" choice of localization. In our case, we use a simpler idea for the Monte Carlo estimators, we include only the $95 \%$ probability trajectories. Formally speaking, for a fixed $S_{s}^{i}=x_{i}$, we take into account only the trajectories associated to $S_{t}^{i}$ that could occur with probability $95 \%$. The errors induced by this truncation are checked to be smaller than ones associated to the Monte Carlo simulation (included in the $95 \%$ confidence interval).

\subsection{MCM accuracy for CVA and CVA sensitivities}

First, we need to point out that we were able to perform our accuracy study thanks to a parallel GPU ${ }^{12}$ implementation on an Nvidia 480 GTX card. Indeed, one of the advantageous of MCM algorithm is its suitability to parallel architecture. The goal of this section is to prove the good accuracy of even a basic implementation of MCM without reducing the variance using the backward conditional density introduced in section 3.2. More advanced numerical study of the backward conditional density will be performed in the future.

We present two parts of the accuracy study: The first one compares MCM to the linear regression method to compute $(6)$ when $1_{\tau \in\left(t_{k}, t_{k+1}\right]}$ is assumed to be equal to 1 and $V_{t_{k}}$ is the price of each contract associated to each payoff specified in Table 1. In the second part, we study the MCM accuracy for both computing the CVA and its sensitivity for the intensity model (38) and the structural model (39).

When considering the values given by the square Monte Carlo (MC2) as the real values, according to Table 1, the values obtained by MCM are almost always better than regression method (Reg). In cases when MCM is less accurate, it is sufficient to increase the number of simulated trajectories. This fact is not true for regression methods because they require the increase of the cardinal of the regression family. Also unlike regression methods, the accurate results obtained by MCM allows to

\footnotetext{
${ }^{10}$ Because of $W^{0}$.

${ }^{11}$ Or added with a much smaller weight.

${ }^{12}$ Graphic Processing Unit/ Central Processing Unit
} 
compute $\Delta$ sensitivity either by using the finite difference (FD) of two MCM prices or by simulating $R_{s, t}^{i}[f](x)$ introduced in (20).

Table 1: Some examples of simulated CVA for each payoff: MCM ( $2^{18}$ trajectories), regression Reg ( $2^{18}$ trajectories), square Monte Carlo MC2 (2 $2^{14}$ trajectories) and $N=5$.

\begin{tabular}{|c|c|c|c|c|c|c|}
\hline Payoff & \multicolumn{6}{|c|}{$d=2$} \\
\hline & \multicolumn{3}{|c|}{$\alpha=0$} & \multicolumn{3}{|c|}{$\alpha=0.5$} \\
\hline & $\mathrm{MCM}$ & Reg & MC2 & $\mathrm{MCM}$ & Reg & MC2 \\
\hline Call on average & 11.4 & 13.1 & 11.8 & 12.7 & 14.6 & 12.8 \\
\hline Put on average & 2.15 & 1.90 & 2.16 & 3.13 & 2.74 & 3.19 \\
\hline Call on max & 21.4 & 24.4 & 22.2 & 19.3 & 22.1 & 19.8 \\
\hline Put on min & 7.07 & 6.97 & 7.28 & 6.28 & 5.81 & 6.37 \\
\hline$\left(\bar{S}_{T}^{2}-S_{T}^{1}\right)_{+}$ & 15.7 & 18.9 & 16.9 & 13.2 & 16.2 & 14.9 \\
\hline$\left(S_{T}^{1}-\bar{S}_{T}^{2}\right)_{+}$ & 5.46 & 7.24 & 6.04 & 3.92 & 4.65 & 3.87 \\
\hline \multirow[t]{3}{*}{ Payoff } & \multicolumn{6}{|c|}{$d=3$} \\
\hline & \multicolumn{3}{|c|}{$\alpha=0$} & \multicolumn{3}{|c|}{$\alpha=0.5$} \\
\hline & $\mathrm{MCM}$ & Reg & $\mathrm{MC} 2$ & $\mathrm{MCM}$ & Reg & $\mathrm{MC} 2$ \\
\hline Call on average & 9.6 & 11.2 & 10.0 & 13.3 & 13.3 & 11.8 \\
\hline Put on average & 2.19 & 1.42 & 1.78 & 3.24 & 2.71 & 3.12 \\
\hline Call on $\max$ & 27.4 & 30.2 & 27.9 & 26.4 & 26.0 & 24.7 \\
\hline Put on min & 11.4 & 9.78 & 9.98 & 9.31 & 7.40 & 8.43 \\
\hline$\left(\bar{S}_{T}^{3}-\frac{S_{T}^{1}+S_{T}^{2}}{2}\right)_{+}$ & 17.6 & 16.8 & 15.8 & 15.7 & 14.9 & 14.1 \\
\hline$\left(\frac{S_{T}^{1}+S_{T}^{2}}{2}-\bar{S}_{T}^{3}\right)_{+}$ & 4.62 & 5.00 & 3.71 & 3.27 & 3.37 & 2.66 \\
\hline
\end{tabular}

Table 2: Some examples of CVA and sensitivity computations (2 $2^{18}$ trajectories).

\begin{tabular}{|c|c|c|c|c|}
\hline Payoff & \multicolumn{4}{|c|}{ Intensity model } \\
\hline & \multicolumn{2}{|c|}{$\mathrm{CVA}_{0, T}$} & \multicolumn{2}{|c|}{$\partial_{S_{0}^{1}} \mathrm{CVA}_{0, T}$} \\
\hline & MC2 & $\mathrm{MCM}$ & using (20) & using FD \\
\hline$\left(S_{T}^{1}-S_{T}^{2}\right)_{+}$ & 1.37 & 1.52 & 0.10 & 0.13 \\
\hline$\left(S_{T}^{1}+S_{T}^{2}-K\right)_{+}$ & 1.92 & 2.12 & 0.06 & 0.07 \\
\hline Call on max & 3.66 & 4.00 & 0.09 & 0.10 \\
\hline Payoff & \multicolumn{4}{|c|}{ Structural model } \\
\hline & \multicolumn{2}{|c|}{$\mathrm{CVA}_{0, T}$} & \multicolumn{2}{|c|}{$\partial_{S_{0}^{1}} \mathrm{CVA}_{0, T}$} \\
\hline & MC2 & $\mathrm{MCM}$ & using (20) & using FD \\
\hline$\left(S_{T}^{1}-S_{T}^{2}\right)_{+}$ & 0.85 & 0.94 & 0.05 & 0.06 \\
\hline$\left(S_{T}^{1}+S_{T}^{2}-K\right)_{+}$ & 0.59 & 0.48 & 0.03 & 0.02 \\
\hline Call on max & 1.17 & 1.18 & 0.09 & 0.12 \\
\hline
\end{tabular}


Once more, according Table 2, when the number of simulated trajectories is sufficient, we obtain accurate values of the CVA and its sensitivity for both intensity and structural modelling framework. Based on what is discussed before, we summarize the comparison of the three methods in the following table

Table 3: Comparison of the different algorithms

\begin{tabular}{|c|c|c|c|}
\hline & $\begin{array}{c}\text { Monte Carlo } \\
\text { square }\end{array}$ & $\begin{array}{c}\text { Monte Carlo } \\
\text { with regression } \\
\text { Complexity }\end{array}$ & $\begin{array}{c}\text { Monte Carlo with } \\
\text { Malliavin calculus }\end{array}$ \\
\hline Parallelization & Easy & Very limited & $O\left(C_{d}^{2} N^{2}\right)$ \\
$O\left(\left(\mathcal{T} d+K^{2} N\right) M N\right)$ & Possible \\
\hline American Op. & No & Yes & Yes \\
\hline Dimensions & Unlimited! & Very limited & Limited \\
\hline Delta & Expensive & Not possible & Limited \\
\hline
\end{tabular}

\section{References}

[1] L. A. Abbas-Turki, S. Vialle, B. Lapeyre and P. Mercier, Pricing derivatives on graphics processing units using Monte Carlo simulation, Concurrency and Computation: Practice and Experience, 2012.

[2] L. A. Abbas-Turki and B. Lapeyre, American Options by Malliavin Calculus and Nonparametric Variance and Bias Reduction Methods, SIAM Journal on Financial Mathematics, 3(1): 479-510, 2012.

[3] V. Bally, L. Caramellino and A. Zanette, Pricing American options by Monte Carlo methods using a Malliavin calculus approach, Monte Carlo Methods and Applications, 11: 97-133, 2005.

[4] T. R. Bielicki, M. Jeanblanc and M. Rutkowski, Credit Risk Modeling, Center of the study of finance and Insurance, Osaka University, Japan.

[5] T. R. Bielecki and M. Rutkowski, Credit Risk: Modelling, Valuation and Hedging. Springer- Verlag, Berlin Heidelberg New York, 2002.

[6] F. Black and J. C. Cox, Valuing corporate securities: Some effects of bond indenture provisions, Journal of Finance, 31: 351-367, 1976.

[7] A. Boukhobza and J. Maetz, CVA, Wrong Way Risk, Hedging and Bermudan Swaption, August 2012.

[8] D. Brigo and F. Mercurio, Interest Rate Models - Theory and Practice: With Smile, Inflation and Credit, Springer Finance, 2007.

[9] D. Brigo, M. Morini and A. Pallavicini, Counterparty Credit Risk, Collateral and Funding: With Pricing Cases For All Asset Classes, John Wiley and Sons, 2013. 
[10] G. Cesari \& al, Modelling, Pricing and Hedging Counterparty Credit Exposure, Springer Finance, 2009.

[11] J. Hull and A. White, CVA and Wrong Way Risk, Financial Analysts Journal, 68(5): 58-69, 2012.

[12] http://www.quantize.maths-fi.com/gaussian_process_database.

[13] R. Merton, On the pricing of corporate debt: The risk structure of interest rates, Journal of Finance, 3:449-470, 1974.

[14] M. Pykhtin, Modeling credit exposure for collateralized counterparties, The Journal of Credit risk, 5(4): 3-27, Winter 2009/10.

[15] D. Rosen, D. Saunders, CVA and wrong way, Journal of Risk Management in Financial Institutions, 5(3): 353-372, 2012.

[16] V. Bally and G. Pagès, A Quantization Algorithm for Solving Multidimensional Discrete-Time Optimal Stopping Problems, Bernoulli, 9(3): 1003-1049, 2003.

[17] P. Glasserman and B. Yu, Number of Paths Versus Number of Basis Functions in American Option Pricing, The Annals of Applied Probability, 14(4): 20902119, 2004.

[18] W. H. Press, S. A. Teukolsky, W. T. Vetterling and B. P. Flannery, Numerical Recipes in $\mathrm{C}++$ : The Art of Scientific Computing, Cambridge University Press, 2002.

[19] G. Pagès and J. Printems, Functional quantization for numerics with an application to option pricing, Monte Carlo Methods and Applications, 11(11): 407446, 2005.

[20] B. Bouchard, I. Ekeland and N. Touzi, On the Malliavin approach to Monte Carlo approximation of conditional expectations, Finance and Stochastics, 8, 45-71, 2004. 